\title{
Intelligent Waste Separator and Recycling (IWSR) using IoT and Evolutionary Fuzzy Logic Technology with energy efficient sensing during Hajj and Umrah crowd seasons
}

\author{
Salma Mahgoub Gaffer Elhag ${ }^{1}$ \\ Assistant Professor, Information Systerns Department, \\ Faculty of Computing and Information Technology, Jeddah, Saudi Arabia ${ }^{1}$
}

\begin{abstract}
This project proposes a prototype of an Intelligent Waste Separator and Recycling (IWSR) that consists of a common trash can, with more containers inside it, using IoT and Evolutionary Fuzzy logic Technology. People can throw their waste, no matter what kind, into the system. The latter can decide what kind of waste it belongs to and to deposit it in the correct container. On the other hand, the system will deploy the IoT technology to manage the crowd of roads during hajj period. The vehicles should be connected to the smart trash cans and to monitoring system to facilitate and affectionate the process of trash gathering and management. The system will rely upon solar energy sensing to be energy efficient.
\end{abstract}

Keywords: Intelligent Waste Separator and Recycling (IWSR), Information and Communication Technologies (ICT), Internet of Things, Geographic Information Systems (GIS), Genetic Fuzzy Optimization

\section{INTRODUCTION}

The technological revolution that has taken place in recent decades, driven by advances and developments in Information and Communication Technologies (ICT) has revolutionized the way people communicate, work, travel, live, etc. Cities need to evolve towards intelligent dynamic infrastructures that serve citizens fulfilling the criteria of energy efficiency and sustainability (1).Various cities around the world have approached the smart city. Each of them usually faced different challenges and prioritized alternative objectives, such as improvement of local everyday life; development of knowledge-based societies; narrowness of the digital divide; and promotion of e-Government locally. Others emphasized on the enhancement of e-commerce services and on local growth, while recently the environmental protection has been put first on the objectives' list (2).As an important part of a smart city, smart environmental sanitation relies on the Internet of Things (IoT) and mobile Internet of Things to realize real-time sanitation management, involving the aspects of human beings, cars and almost everything. Also, it provides rational plans for sanitation management modes and utilizes digital technology to improve sanitation operations (3).

Waste gathering is one of the most important public services in any modern city (3). Smart Waste Management tries to solve this challenge with the help of techniques such as Internet of Things, machine learning and cloud computing (4).Waste gathering becomes very urgent in the period of Hajj ,so This task needs to be facilitated . Most municipalities have a fixed schedule with fixed routes for collecting garbage, and this can result in various inefficiencies: sometimes garbage trucks pick up trash cans that were almost empty, and sometimes overflowing cans are left in this state for days. These problems can be overcome by making the trash cans Intelligent, i.e. placing a sensor in each can to detect the garbage fill level, and periodically sending this data to a central server: in this way, waste gathering schedules and routes can be optimized based on the actual need, increasing the level of service and decreasing costs. The IoT tracking help operation managers to understand the optimal times for waste collections and even recommends the optimal collection routes. The system optimizes waste collection routes and ultimately reduces fuel consumption. It allows trash collectors to plan their daily/weekly pick and hence help to mange the crowd problem. Besides assisting with the optimal collection routes, the software also predicts collection times by utilizing historical data

Since clean-tech, Internet of Things and the environmental issues are important topics; there are several products competing in similar commercial niches. The list of commercial products can be divided into two types, retrofitted sensors and smart containers. Smart containers are special-made containers, e.g., urban dust bins or containers for cardboard which measure the level while mechanically compressing the cardboard. A smart bin is a waste or recycling bin outfitted with a sensor that can detect bin fill level, collection events, fire, tilt and temperature. There are a variety of sensors on the market that use ultrasonic sensors, laser measurement and image recognition to collect data. The containers or dustbins are typically smaller in size and target restaurants, industries, and cities. Examples of such 


\title{
International Advanced Research Journal in Science, Engineering and Technology
}

\author{
Vol. 6, Issue 2, February 2019
}

products are Big Belly and Clean CUBE by Ecube .Retrofitted sensors are the same type of system as Smart Recycling $\mathrm{R}$ and examples of such products are Enevo, CleanFLEX by Ecube, Sensoneo, Onsense, Smart Waste by Citibrain and Smart Bin . Out of these solutions CleanFLEX seems to be the most relevant to this project since they also offer predictive analysis of the filling level. CleanFLEX is a wireless ultrasonic fill-level sensor that securely monitors a container's fill-level and sends real-time data to CleanCityNetworks, our cloud-based monitoring and data analytics platform. CleanFLEX can be easily attached to any type of container or tank to monitor any type of waste, including solids and liquids. Together with the cloud platform, CleanFLEX enables cities and waste management companies to increase their operational waste collection efficiency by up to $50 \%$. The software platform visualizes the capacity of each trash bin in traffic light system: green color means that the waste container has still plenty of space, and red color gives a signal to the operation manager that collection is required.

\section{PRELIMINARIES}

\section{A. Intelligent Waste Separation systems}

To get rid of Waste in an efficient and environment-friendly way is an absolute a necessity service for Smart Cities The way to manage waste(5) become a problem due to the way people get rid of it. Most of garbage is buried or burnt or even kept in places to which it does not belong. Big volumes of garbage thrown away and the methods used to store it cause air, water, and soil pollution. Furthermore, in crowded place like Hajj and Umrah it becomes more necessary to find intelligent efficient way collect and get rid of waste with (6) out to make the traffic problem worse. to the transportation. Fortunately, people can count on other methods to reduce the quantity of produced litter. An answer is recycling by re-using the materials (7). Currently, the traditional way to separate waste is to use different containers for each kind of waste separating trash manually, which does not always work.

There are a lot of trials to automate the process of waste classification since early $1990 \mathrm{~s}(8)(9)(10)(11,12)$.The aim of this paper is to present an Intelligent Waste Separator (IWS) which can replace the traditional way of dealing with waste; the proposed device receives the incoming waste and places it automatically in different containers by using a multimedia embedded processor, image processing, and machine learning in order to select and separate waste. Furthermore, the system uses a smart frame work to simulate a network of smart bins to facilitate the collection of waste in the crowd of Hajj or Umrah seasons.

\section{B. Internet of Things Technology}

Each day, cities around the world are investing more and more to become smarter (13). A Smart City is a city well performing in a forward-looking way in the following fundamental components (i.e., Smart Economy, Smart Mobility, Smart Environment, Smart People, Smart Living, and Smart Governance), built on the 'smart' combination of endowments and activities of self-decisive, independent and aware citizens(14). Internet of Things (IoT) Technology can be applied both in ITS and Smart cities forming an advanced platform for novel applications various systems and application in the field of Smart Cities and Intelligent Transportation Systems (5). Although there are a lot of challenge waste managements is one of the priories in any IoT enabled smart cities(15).

This paper practically demonstrates how Internet of Things (IoT) integration with data access networks, Geographic Information Systems (GIS), genetic fuzzy optimization, and electronic engineering can contribute to improve cities' management systems. We present a waste collection solution based on providing intelligence to trashcans, by using an IoT prototype embedded with sensors, which can read, collect, and transmit trash volume data over the Internet

\section{Evolutionary Fuzzy rule base classification systems}

The use of Artificial Intelligence and Computational intelligence is now common to solve a lot of classification and other data mining problems (16). The automatic definition of a Fuzzy Rule Based Systems FRBS can be seen as an optimization or search problem (17). In this respect, Evolutionary algorithms EAs are a well-known and widely used global search technique with the ability to explore a large search space for suitable solutions only requiring a performance measure (18).

In addition to their ability to find near optimal solutions in complex search spaces, the generic code structure and independent performance features of EAs make them suitable candidates to incorporate a priori knowledge (19).

EAs codify the required solutions in terms of strings of genes (represented as numbers) or chromosomes. Each chromosome is associated to one or more fitness functions (quality of the solution) and evolved by using concepts 


\title{
International Advanced Research Journal in Science, Engineering and Technology
}

\author{
Vol. 6, Issue 2, February 2019
}

inspired to the evolutionary process, such as mutation and crossover. The new individuals (chromosomes) that are obtained at each step are evaluated with respect to the fitness functions and the best individuals are promoted. Therefore, EAs perform an exploration of a certain search space, related with the structure codified into the individual. In the case of FRBSs, this a priori knowledge encoded into the individuals may be in the form of linguistic variables, fuzzy membership function parameters, fuzzy rules, number of rules, etc. These capabilities extended the use of EAs in the development of a wide range of approaches for designing FRBSs over the last few years. In Figure 1, the evolutionary process for learning and/or tuning different components of an FRBCS is presented.

one of the most popular hybridizations is possibly the one between Fuzzy Rule Based Systems FRBSs and Evolutionary Computation leading to EFSs (20)

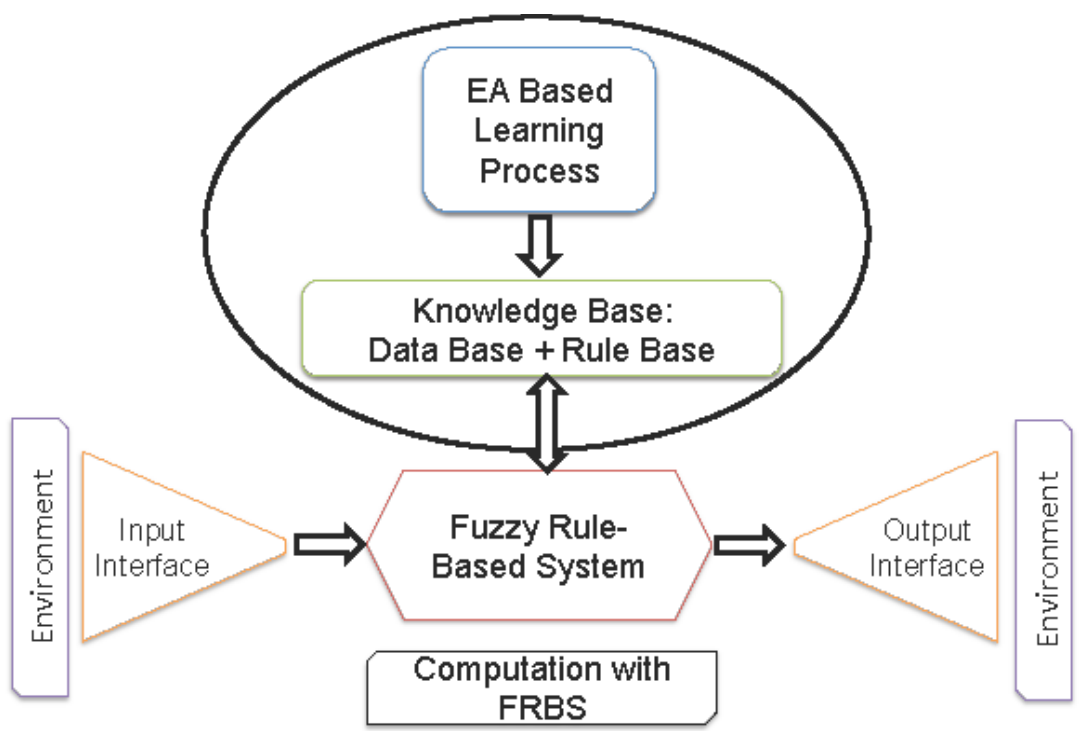

Figure 1 Evolutionary Fuzzy System

In this work, we prose to build a classifier model base on Evolutionary Fuzzy Systems which should be able to classify different kind of waste automatically.

\section{PROPOSAL OBJECTIVES}

- $\quad$ To Solve the problem of Waste management during Hajj and Umrah

- $\quad$ To help deal with the Problem of Crowd management through optimizing waste trucks movement

- $\quad$ To help to enhance fuel consumption through optimizing waste collection using the smart system

- To be energy efficient by using solar energy sensing

- To improve cities’ management systems through Smart cities Strategies

\section{METHODOLOGY}

Formulation of hypotheses by proposing the Evolutionary Fuzzy logic classifier to enhance waste classification Implementation of hypothesis and the development of the software over the network simulator Testing and comparison of the results obtained against the already existing techniques Modification of the initial hypotheses, considering the obtained results: the initial methods can be modified and finely tuned in this phase to achieve better performance.

\section{PROPOSED SYSTEM DEVELOPMENT}

Our System consists of two sub-systems including a hardware system and a software system. The hardware system is of a trash bin framework based on the core module Raspberry Pi and the software one is of an image classification algorithm platform based on SURF-BoW algorithm and multi-class evolutionary fuzzy system classifier. In our experiment, the images produced during training and testing are both obtained from webcam in our system and extra processing with affine transformation and noise-adding operation. The experimental results show that among the five categories of waste, the battery waste performs best with $100 \%$ classification accuracy. Besides, the average 


\section{International Advanced Research Journal in Science, Engineering and Technology}

Vol. 6, Issue 2, February 2019

classification accuracy is up to $83.38 \%$. Therefore, our system has reliable practicability and robustness, which is expected to be applied to deal with the waste sorting problems in our daily life.

A. Raspberry (Smart Trsh Can device ) with sensor : A Model Based on Fuzzy logic Evolutionary Algorithms, that classifiy the waste to one of 5 classes using image recognition [21]

B. ONLINE OPTIMAL-ROUTING-TRACKING SYSTEM BASED OF IOT

We will use network simulator IoTIFY https://iotify.help/network/smart-city/waste-mgmt.html to simulate our network of trash cans. IoTIFY network simulator can be used to simulate any IoT device, including trash cans. (22)

\section{MODEL BUILDING}

We stated building our network of trash cans by adding nodes to the network simulator Figure 2.

\begin{tabular}{l} 
ICES (View All) \\
\hline arch by name or tags \\
\hline Name items \\
TrashCan001 \\
TrashCan002 \\
TrashCan003
\end{tabular}

Figure 2 Iotify Trashcan network

$$
\leftrightarrow \text { LOS AN T Dashboards - Applications : }
$$

\section{NEW DASHBOARD}

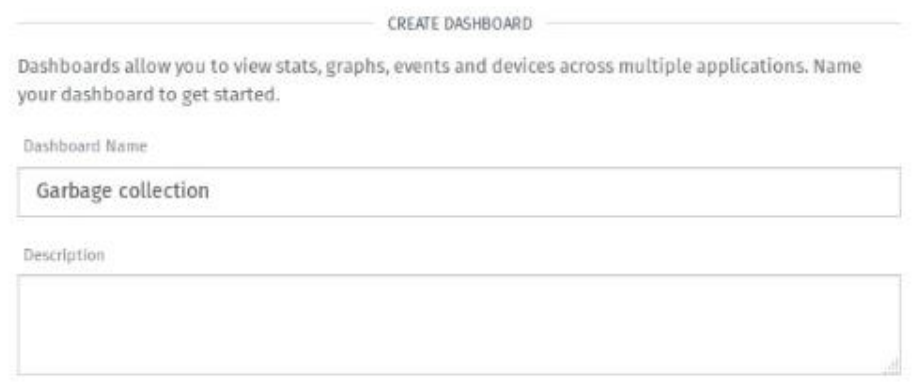

Figure 3 System Dashboard

The nodes represent devices which in our case are standalone devices that are connected and report their status directly to the system. Each trash can node is a Raspberry (Smart Trash Can device) with sensor. The network simulator allows creating personalized dashboards to display data coming from any device in a given application, and also to send commands to a device. A dashboard is created to monitor the status of the 3 trash cans we created in the model to empty the trash cans.

\section{TESTING}

When testing the system there are different views to show the amount of trash in each can.There are also a GPS history to show one or more devices over a time period.A map view is thetre with the location of the trash cans and a bar chart indicating the garbage level in each trash can Figure 4.The system also shows GPS map with smart trash cans and Garbage Level of each can Figure 5. 


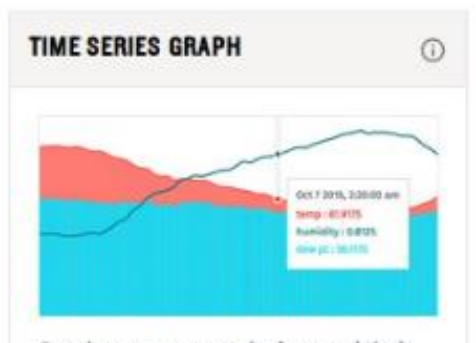

Graphs one or more devices and their attributes over a custom time range.

\section{Customize}
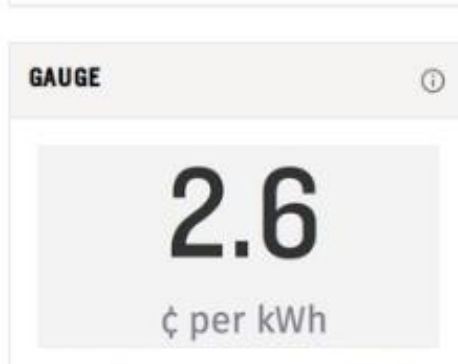

Displays the value of a single attribute.

Customize

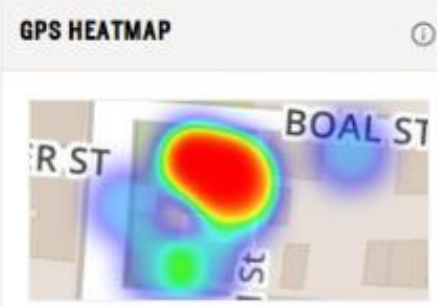

Heatmap of the GPS positions of one or more devices at any point in time.

\section{Customize}

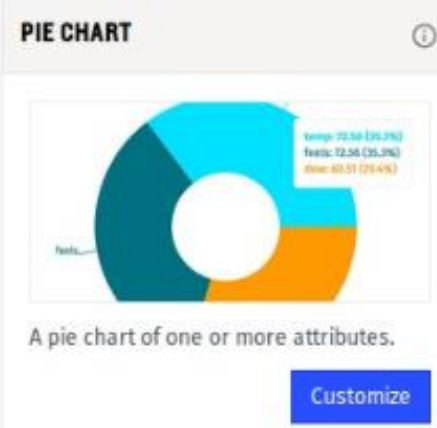

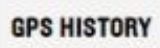

(1)

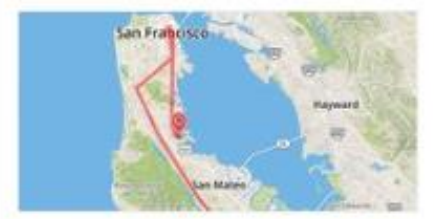

Displays the GPS history of one or more devices over a time period.

\section{Customize}

BAR CHART

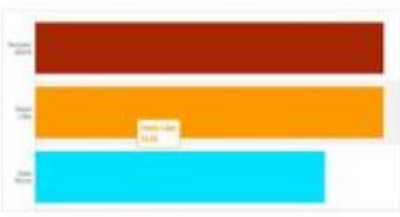

A bar chart of one or more attributes.

Customize

Figure 4 Different charts of the smart cans with different attributes
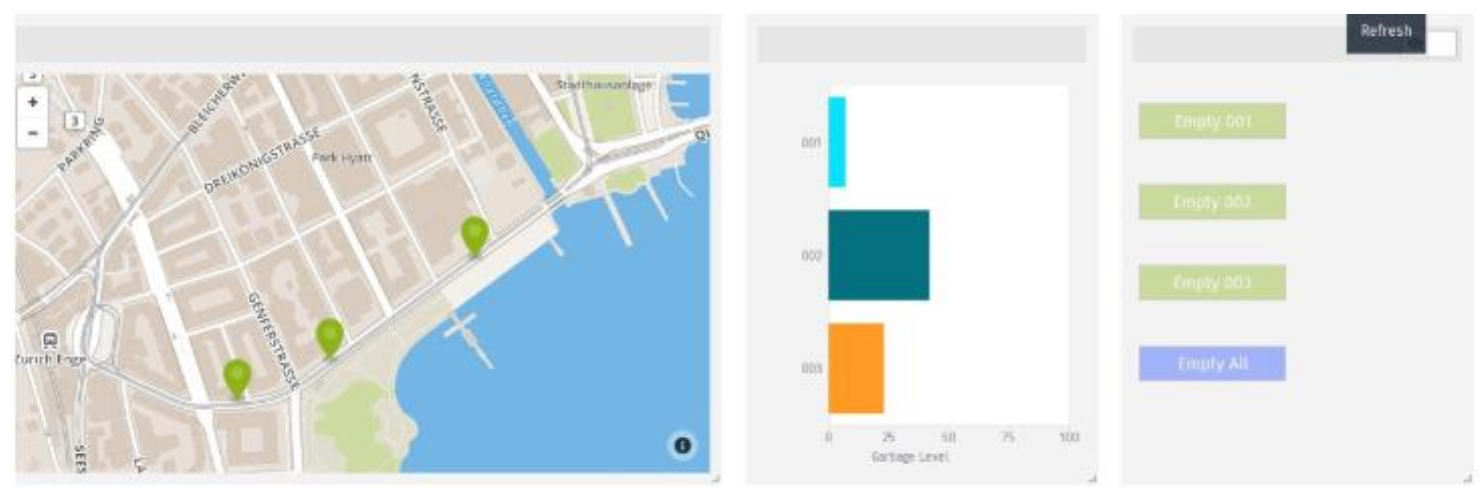

Figure 5 GPS map with smart trash cans and Garbage Level of each can

\section{RESULTS AND DISCUSSION}

This study found that the process of waste management during hajj is an open area for research and can be optimized by using IoT technology as a smart city technology in synergy with Evolutionary Fuzzy classification sysem. The study provided initial results that are promising with regard to enhanced trash trucks road consumption which will eventually help to eliminate crowd during crowd seasons of hajj and Umrah. This can help to enhance fuel consumption as trucks will only drive when getting signals during the network from empty waste trash cans. The proposed system is meant to be to be energy efficient by using solar energy sensing.

\section{CONCLUSION}

The system optimizes waste collection routes and ultimately reduces fuel consumption. It allows trash collectors to plan their daily/weekly pick and hence help to manage the crowd problem. Besides assisting with the optimal collection routes, the software also predicts collection times by utilizing historical data. The data regarding the garbage levels will be used to provide optimized route for garbage collecting vans, which will reduce the crowd problem during hajj and 


\title{
International Advanced Research Journal in Science, Engineering and Technology
}

\author{
Vol. 6, Issue 2, February 2019
}

also reduce cost associated with fuel which will means saving energy. According to this conclusion and to the results listed in the results section we would recommend the following:

It is necessary to apply studies that are based on modeling and simulation using computers to evaluate the proposals as modeling and simulation provide:

- Implement and evaluate the effectiveness of the proposed system and identify their advantages and disadvantages in real network.

- Work in accordance with stakeholders and decision makers that helps in evaluating the development plans to be implementing the proposed system and simulated network.

- Conduct more studies to detect possible obstacles that are not considered during this initial phase, and the possibility of improving the solutions that reduce the effects of obstacles, which can be avoided if foreseen using modeling and simulation.

- Conduct feasibility study and Cost-Benefit Study for the hall system Development and implementation.

\section{REFERENCES}

[1]. Pellicer S, Santa G, Bleda AL, Maestre R, Jara AJ, Skarmeta AG, editors. A global perspective of smart cities: A survey2013: IEEE.

[2]. Anthopoulos L, Fitsilis P. Using Classification and Roadmapping techniques for Smart City viability's realization. Electronic Journal of eGovernment. 2013;11(2).

[3]. Chen AH, Warren J. Sustainable Growth for China: When Capital Markets and Green Infrastructure Combine. Chinese Economy. 2011;44(5):86-103.

[4]. Rutqvist D. Data-Driven Emptying Detection for Smart Recycling Containers. 2018.

[5]. Medvedev A, Fedchenkov P, Zaslavsky A, Anagnostopoulos T, Khoruzhnikov S, editors. Waste management as an IoT-enabled service in smart cities. Conference on smart spaces; 2015: Springer.

[6]. Mahajan S, Kokane A, Shewale A, Shinde M, Ingale S. Smart Waste Management System using IoT. International Journal of Advanced Engineering Research and Science.4(4).

[7]. Torres-García A, Rodea-Aragón O, Longoria-Gandara O, Sánchez-García F, González-Jiménez LE. Intelligent waste separator. Computación y Sistemas. 2015;19(3):487-500.

[8]. Douglas JM. Process synthesis for waste minimization. Industrial \& engineering chemistry research. 1992;31(1):238-43.

[9]. Chen G, Hung W, Chang I, Lee S, Lee D. Continuous classification of moisture content in waste activated sludges. Journal of Environmental Engineering. 1997;123(3):253-8.

[10]. LaGrega MD, Buckingham PL, Evans JC. Hazardous waste management: Waveland Press; 2010.

[11]. Zanella A, Bui N, Castellani A, Vangelista L, Zorzi M. Internet of things for smart cities. IEEE Internet of Things journal. 2014;1(1):22-32.

[12]. Haines-Young R, Potschin M. Common international classification of ecosystem services (CICES, Version 4.1). European Environment Agency. 2012;33

[13]. Asimakopoulos G, Christodoulou S, Gizas A, Triantafillou V, Tzimas G, Viennas E, et al., editors. Towards a dynamic waste collection management system using real-time and forecasted data. Proceedings of the 16th international conference on engineering applications of neural networks (INNS); 2015: ACM.

[14]. Giffinger R, Fertner C, Kramar H, Meijers E. City-ranking of European medium-sized cities. Cent Reg Sci Vienna UT. 2007:1-12.

[15]. Anagnostopoulos T, Zaslavsky A, Kolomvatsos K, Medvedev A, Amirian P, Morley J, et al. Challenges and opportunities of waste management in IoT-enabled smart cities: a survey. IEEE Transactions on Sustainable Computing. 2017;2(3):275-89.

[16]. Ward DJ, MacKay DJ. Artificial intelligence: fast hands-free writing by gaze direction. Nature. 2002;418(6900):838

[17]. Balasubramanian SV. Embedded System for Waste Management using Fuzzy Logic.

[18]. Park W, Lee H-K, editors. Fuzzy Logic Based Satellite Image Classification: Generation of Fuzzy Membership Function and Rule from Training Set. MVA; 1998: Citeseer.

[19]. Pena-Reyes CA, Sipper M. A fuzzy-genetic approach to breast cancer diagnosis. Artificial intelligence in medicine. 1999;17(2):131-55.

[20]. Cordón O, Herrera F, Hoffmann F, Magdalena L. Evolutionary tuning and learning of fuzzy knowledge bases: Vol. 19 of Advances in Fuzzy Systems-Applications and Theory. Singapore: World Scientific; 2001.

[21]. Liu Y, Fung K-C, Ding W, Guo H, Qu T, Xiao C. Novel Smart Waste Sorting System based on Image Processing Algorithms: SURF-BoW and Multi-class SVM. Computer and Information Science. 2018;11(3):35.

[22]. Shuan W, Haizhou A, Kezhong H. Difference image based multiple motion targets detection and tracking [J]. Journal of Image and Graphics. $1999 ; 6$. 